\title{
The Role of Cardiovascular Magnetic Resonance in Sports Cardiology; Current Utility and Future Perspectives
}

\section{Emmanuel Androulakis, MRCP MSC PhD ${ }^{1}$} Peter P. Swoboda, MRCP, $P h D^{2, *}$

\author{
atates \\ ${ }^{1}$ St George's University Hospitals NHS Foundation Trust, London, UK \\ ${ }^{*}, 2$ Leeds Institute of Cardiovascular and Metabolic Medicine, University of Leeds, \\ Clarendon Way, Leeds, LS2 9JT, UK \\ Email: p.swoboda@leeds.ac.uk
}

Published online: 31 August 2018

(C) The Author(s) 2018

This article is part of the Topical Collection on Sports Imaging

Keywords Cardiac magnetic resonance imaging $\cdot$ Sports cardiology $\cdot$ Fibrosis $\cdot$ Cardiomyopathies

Abbreviations CMR Cardiovascular magnetic resonance $\cdot E C V$ extracellular volume $\cdot L V$ left ventricle

\begin{abstract}
Purpose of review Cardiovascular magnetic resonance (CMR) is frequently used in the investigation of suspected cardiac disease in athletes. In this review, we discuss how CMR can be used in athletes with suspected cardiomyopathy with particular reference to volumetric analysis and tissue characterization. We also discuss the finding of nonischaemic fibrosis in athletes describing its prevalence, distribution and clinical importance.

Recent findings The strengths of CMR include high spatial resolution, unrestricted imaging planes and lack of ionizing radiation. Regular physical exercise leads to cardiac remodeling that in certain situations can be clinically challenging to differentiate from various cardiomyopathies. Thorough morphological assessment by CMR is fundamental to ensuring accurate diagnosis. Developments in tissue characterization by late gadolinium enhancement and T1 mapping have the potential to be powerful additional tools in this challenging clinical situation. Using late gadolinium enhancement, it is also possible to detect non-ischaemic fibrosis in athletes who do not have overt cardiomyopathy. The mechanisms of this fibrosis are unclear; however, it does appear to be clinically important. We also review data on the prevalence of non-ischaemic fibrosis in athletes.
\end{abstract}


Summary CMR is a powerful tool to aid in the diagnosis of cardiomyopathy in athletes. It may also have a future role in assessing fibrosis related to long-term participation in sport.

\section{Introduction}

Long-term exercise leads to structural and functional cardiac adaptation often termed 'athlete's heart'. Typical features include left ventricular hypertrophy (LVH), left ventricle (LV) and right ventricle (RV) cavity dilatation and associated electrocardiographic (ECG) changes [1, 2]. Athlete's heart facilitates an increase in stroke volume and cardiac output as a physiological response to exercise training and may potentially vary across different types of exercise [3].

In clinical practice, it can be challenging to differentiate the physiological changes of athlete's heart from cardiomyopathy, and in this review article, we will discuss both established and novel cardiovascular magnetic resonance (CMR) techniques that may be helpful for this purpose. Cardiomyopathy is one of the leading causes of death in athletes and often once this diagnosis is made precludes participation in competitive sport. $\mathrm{CMR}$, therefore, has a potentially vital role in improving diagnosis [4].

Recent evidence suggests that prolonged participation in certain sports may predispose certain individuals to cardiac fibrosis, which may be associated with increased risk of arrhythmias, particularly atrial fibrillation $[5,6]$.

In this systematic review, we discuss the role of CMR in diagnosing cardiomyopathy in athletes and in particular review data on cardiac fibrosis.

\section{CMR assessment of athlete's heart}

Echocardiography is the first-line investigation to assess cardiac morphology in athletes given its low cost, widespread availability and lack of ionizing radiation. CMR is increasingly used in clinical practice as it allows accurate visualization and quantification of the heart in any plane without limitation by acoustic windows. Using CMR imaging, it is also possible to make an assessment of myocardial tissue characteristics including fat and water content, fibrosis and myocyte mass. By acquiring a stack of short-axis images with no interslice gap with full ventricular coverage, it provides the opportunity to inspect the entire LV myocardium for abnormalities, including focal hypertrophy or regional wall motion abnormalities. Unlike two-dimensional (2D) techniques, cine CMR imaging does not rely on geometric assumptions or calculations based on incomplete sampling of the cardiac volumes [7].

Gadolinium-based CMR contrast agents are exclusively extracellular and can only passively enter damaged cells with a leaky cell membrane. This phenomenon is exploited in late gadolinium enhancement (LGE) CMR imaging. LGE imaging involves administration of typically $0.1-0.2 \mathrm{mmol} / \mathrm{kg}$ of a gadoliniumbased contrast agent. After a delay of 5-20 min, the contrast agent is retained to a greater extent than in areas of scar or fibrosis than in normal myocardium. The pattern of enhancement on LGE can aid in the diagnosis of cardiomyopathy. For example, subendocardial or transmural LGE is typically seen in ischemic cardiomyopathy, mid wall LGE in dilated and hypertrophic cardiomyopathies and subepicardial LGE in myocarditis [8]. 


\section{Defining athlete's heart}

T1 mapping is a method for quantitative assessment of tissue characteristics. The myocardial T1 time can be measured without contrast, native T1, or following the administration of intravenous gadolinium-based contrast agent, post-contrast T1. By combining both these measures, the myocardial extracellular volume (ECV) fraction can be approximated, a value that has been validated histologically in several cardiomyopathies [8].

Morphology of the left ventricle in athlete's heart has primarily been studied by echocardiography with a clear pattern of LV dilatation and hypertrophy being reported (although varying by sporting type) [1]. The improved reproducibility of CMR has meant that it is possible to validate these findings, with a smaller sample size and in longitudinal studies $[9,10,11 \bullet]$. This is exemplified by Arbab-Zadeh et al.[11•] who demonstrated that 1 year of prolonged and intensive endurance training leads to cardiac morphological adaptations in 12 previously sedentary young subjects similar to those observed in elite endurance athletes.

Given the complex structure of the RV morphological assessment by CMR is more reproducible than echocardiography. Increases in RV mass, end diastolic and stroke volumes relative to non-athletes have been described. The ratio of LV to RV size was maintained, leading to the conclusion that athlete's heart syndrome involves balanced remodeling of both ventricles [10].

\section{Differentiating physiology from pathology}

\section{Hypertrophic cardiomyopathy}

Hypertrophic cardiomyopathy (HCM) is the leading cause of sudden cardiac death in young athletes worldwide [12]. Traditional methods for differentiating physiological left ventricular hypertrophy (athlete's heart) from HCM have relied on parameters derived from sedentary HCM patients and healthy athletes. As the leading cause of sudden cardiac death in younger populations, the discrimination of HCM from athlete's heart has immediate clinical relevance [12].

In general, in subjects with good echocardiographic images, CMR provides similar information on ventricular function and morphology but can provide incremental diagnostic value in patients with poor acoustic windows or when some LV regions are poorly visualized - such as the anterolateral wall, the LV apex and the right ventricle $[13,14]$. CMR also has a role in identification of subtle markers of HCM including aneurysms, thrombi, myocardial crypts and papillary muscle abnormalities [15].

Cine CMR can be used to differentiate athlete's heart from pathological LVH using multiple geometric measures. Petersen et al. reported that left ventricular diastolic wall to volume ratio had the highest area under the curve (0.993) providing a sensitivity of $80 \%$ and a specificity of $99 \%$ to distinguish athlete's heart from all forms of pathologic LVH [16].

Echocardiography tends to underestimate LA volumes and when assessed by CMR atrial volumes are significantly larger in endurance athletes than controls [17]. The presence of LA dilatation points more towards a diagnosis of athlete's heart than HCM [18]. 
HCM is associated with mid wall fibrosis detected on LGE imaging predominantly in areas of hypertrophy and is therefore useful in making the diagnosis. LGE is only present in approximately two thirds of patients with HCM and may be even lower in athletes with $\operatorname{HCM}[19,20]$. In an athlete with unexplained LV hypertrophy, the presence of typical scar may aid the diagnosis of HCM but its absence cannot exclude it.

Using T1 mapping, it has been shown that in athletes, as fitness increases there is an increase in myocyte mass, with a relatively constant extracellular

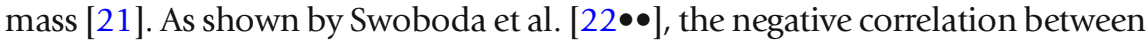
ECV and wall thickness in athletes and sedentary controls suggest that the increase in LV mass in healthy myocardium is mediated by cellular hypertrophy. In HCM, there is a positive correlation between wall thickness suggesting that hypertrophy is mediated by cellular disarray and extracellular matrix expansion. Thus, CMR using T1 mapping has a potential role in the exclusion of HCM in athletes presenting with LV hypertrophy (Fig. 1).

\section{Arrhythmogenic right ventricular cardiomyopathy}

The diagnosis of arrhythmogenic right ventricular cardiomyopathy (ARVC) is based on "Task Force" criteria (TFC) of clinical, histological and electrophysiological and imaging parameters [23]. Imaging criteria require presence of both qualitative findings (RV regional akinesia, dyskinesia, dyssynchronous contraction) and quantitative metrics (decreased ejection fraction or increased indexed RV end-diastolic volume). Major CMR criteria have a sensitivity of 68 to $76 \%$. Minor criteria (RV ejection fraction $40-45 \%$ or indexed RV end-diastolic volume $100-110 \mathrm{~mL} / \mathrm{m}^{2}$ for men and $90-100 \mathrm{~mL} / \mathrm{m}^{2}$ for women) had a higher sensitivity (79 to $89 \%$ ), but a consequently lower specificity (85 to 97\%) [23]. The distal RV (from the moderator band to the apex) shows highly variable contraction patterns and regional wall motion abnormalities in the sub-tricuspid region are more significant [24]. RV scarring detected on LGE is a common

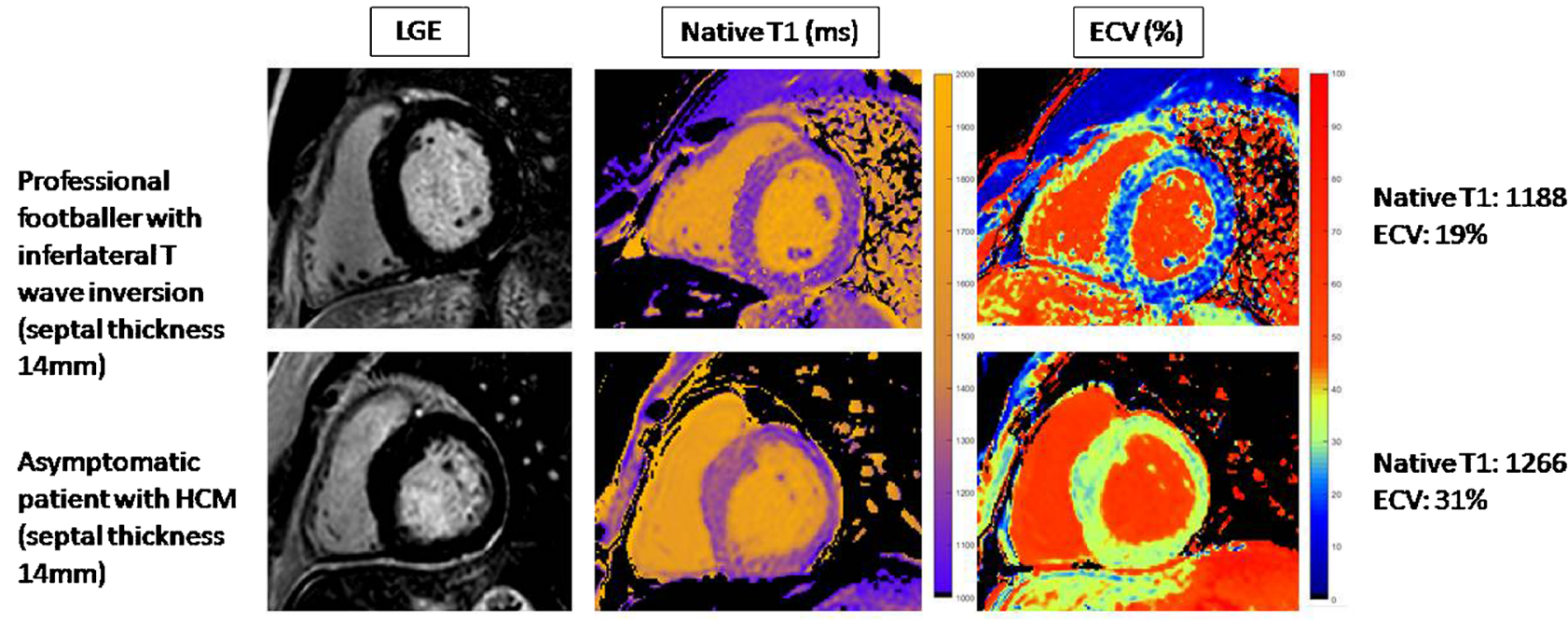

Normal values (Philips 3T): NativeT1 1190+/-50ms; ECV 24-29\%

Fig. 1. CMR using T1 mapping and ECV has a potential role in the exclusion of HCM in athletes presenting with LV hypertrophy. 
finding in ARVC; however, it is limited by the ability to acquire high-quality LGE images of the RV free wall. This technique is therefore not routinely used [25].

Chronic RV abnormalities and acute dysfunction after endurance sporting events have both been described. Heidbüchel et al. [26] proposed the term "exercise-induced right ventricular cardiomyopathy" in reference to the disproportionate rates of $\mathrm{RV}$ dysfunction in elite athletes presenting with serious arrhythmias based on echocardiography, CMR and/or ventriculography. The distinction between ARVC and the effects of exercise are not absolute, and interestingly, exercise may accelerate the development of ARVC in those who are genetically predisposed [27]. On the other hand, a growing body of evidence has suggested that athletes' RV function is in fact superior to non-athletes as reported by D'Andrea et al. [28]. Therefore, although athletes may have impaired RV function immediately after intense endurance exercise and in the context of ventricular arrhythmias, healthy athletes, at rest, should have RV functional measures that are at least as good as the nonathletic population. La Gerche et al. [29•] tested the hypothesis that intense exercise may promote proarrhythmic remodeling in some athletes. Exercise imaging was performed in 17 athletes with RV ventricular arrhythmias, of which eight (47\%) had an implantable cardiac defibrillator (ICD), 10 healthy endurance athletes and seven non-athletes. Echocardiographic as well as CMR measures at rest and during intense exercise combined with invasive measurements of pulmonary and systemic artery pressures were obtained. Among athletes with normal cardiac function at rest, exercise testing revealed RV contractile dysfunction among athletes with RV arrhythmias. This is still a matter of ongoing debate that has potentially been underappreciated. With recent advances in RV imaging CMR may potentially elucidate some of these aspects.

CMR is the reference technique for the quantification of ventricular volumes and functional parameters, to measure wall thickness and ventricular mass in patients with DCM [30]. Evidence suggests a significantly more heterogeneous end-diastolic LV wall thickness in patients with DCM compared to normal population. Also the physiological gradient in systolic wall thickening between LV basal and apical segments disappears with DCM. RV mass has also been shown to be preserved in DCM patients as compared to normal subjects, whereas LV mass is significantly greater with evidence of larger trabeculae as compared to normal subjects [30,31], while in advanced cases, LV dysfunction may be associated with diffuse myocardial wall thinning (diastolic wall thickness $<5.5 \mathrm{~mm}$ ) [30, 31]. LGE has been described as being present in patients with DCM in $12-35 \%$ of the cases, the most common pattern being characterized by a midwall linear distribution which might represent the intramural layer of septal fibrosis which has been observed in pathologic samples [30, 32].

In the most extreme athletic training, cardiac remodeling can be profound. It was shown by means of echocardiography on 286 Tour de France cyclists that more than half of the athletes had LV diastolic dimensions exceeding $60 \mathrm{~mm}$ and $11.7 \%$ had a left ventricular ejection fraction $(\mathrm{LVEF}) \leq 52 \%$. This may pose significant difficulty in differentiating athlete's heart from DCM [33].

If resting measures do not differentiate the athlete from the patients, potentially systolic dysfunction during exercise might be an important aspect. 
Abernethy et al. [34] demonstrated good augmentation of systolic function in professional footballers with low normal LVEF. Some studies in heart failure populations have observed no exercise induced increment in LVEF using echocardiography whereas others reported some degree of augmentation [32].

Claessen et al. [35] have proposed that contractile reserve can be assessed by exercise CMR and to discriminate physiological and pathological LV remodelling impairment of LV ejection fraction.

Both native T1 and ECV have been shown to be elevated in patients with DCM, even in those without midwall fibrosis $[36,37]$. A study comparing early $\operatorname{DCM}(N=16)$ and exercisers with low normal $\operatorname{LVEF}(N=21)$ showed that exercisers had normal native T1 and ECV whereas both were elevated in those with DCM and this difference could be used to discriminate the two groups [38].

Left ventricular non-compaction cardiomyopathy

Non-compaction cardiomyopathy occurs due to an autosomally dominant inherited trait in which the middle and apical segments exhibit a thin compact wall with regional dilatation, dysfunction and significant hyper-trabeculation. There are different definitions including an end-diastolic ratio of noncompacted to compacted LV myocardium of greater than or equal to 2.3. Also, LV wall motion abnormalities, global dysfunction or coronary intraventricular thrombi are often present in the disorder [39].

However recently, an unexpectedly large prevalence of LVNCC has been reported in athletes, raising the question of the appropriateness of current diagnostic criteria [40,41]. In a large athlete population comprising 2501 consecutive athletes who underwent a cardiac evaluation including physical examination, ECG, exercise test and echocardiography and additionally CMR, a marked LV trabecular pattern was seen in $1.4 \%$. Only a small subset of these athletes $(0.1 \%)$ showed familial, clinical and morphologic changes supporting the diagnosis of LVNCC. It is important to note that the majority of athletes with increased trabeculations were not associated with LV dysfunction and/or positive family history, likely representing a morphologic LV variant, of limited clinical significance [42].

\section{CMR coronary imaging}

Anomalous coronary arteries are relatively common cause of death during sport particularly when the coronaries have a malignant interarterial course [4]. The course of the coronary arteries can be depicted by CMR either with or without administration of contrast [43]. CMR coronary imaging does not expose the athlete to ionizing radiation and is therefore an ideal test for young athletes. Coronary magnetic resonance angiography has been used in a study with 335 individuals (including 207 athletes) with a malignant variant of the right coronary artery identified in four subjects [44].

\section{Myocardial fibrosis in athletes}

Several studies have reported myocardial fibrosis (MF) detected by LGE CMR although the prevalence and pattern have varied (Table 1). However, the causes and mechanisms of MF are unclear. Tahir et al. [47••] comparing 83 


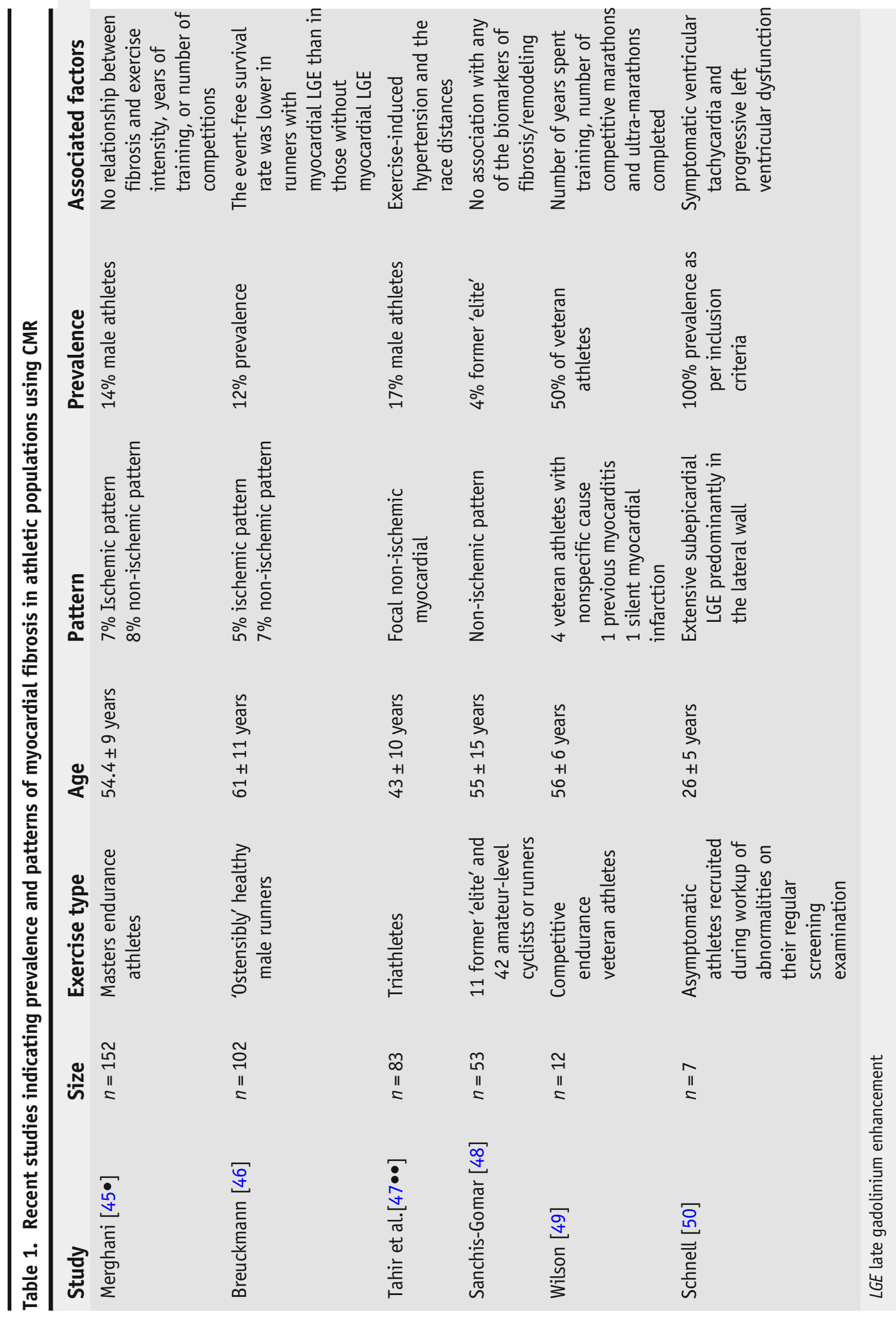


asymptomatic triathletes undergoing $>10$ training h per week and 36 sedentary controls using LGE and extracellular volume (ECV) CMR demonstrated focal nonischemic MF in 9 of $54(17 \%)$ male triathletes but in none of the female triathletes. Fibrosis was associated with exercise-induced hypertension and the race distances. Merghani et al. [45•] assessed 152 master athletes $54.4 \pm$ 8.5 years of age and 92 matched controls with low Framingham 10-year coronary artery disease risk scores in the aim of identifying the prevalence of subclinical coronary artery disease. Most athletes and controls had a normal coronary artery score (CAC) score; however, male athletes had a higher prevalence of atherosclerotic plaques of any luminal irregularity (44.3 versus 22.2\%) compared with sedentary males. Of note, male athletes demonstrated predominantly calcific plaques $(72.7 \%)$, whereas sedentary males showed predominantly mixed morphology plaques $(61.5 \%)$ and the number of years of training was the only independent variable associated with increased risk of CAC. Interestingly, in regards to the CMR findings, 14\% male athletes but none of the controls revealed LGE, half of whom had a pattern consistent with previous myocardial infarction. Earlier, Wilson et al. [49] having examined 12 lifelong veteran male endurance athletes, 20 age-matched veteran controls and 17 younger male endurance athletes without significant comorbidities, demonstrated that in six (50\%) of the veteran athletes, LGE of CMR indicated the presence of myocardial fibrosis and in one case, the pattern was consistent with previous episode of acute myocarditis.

Also, the amount and intensity of exercise training, as well as the lifelong exercise exposure should be taken into account to test the hypothesis that MF prevalence differs between athletes and the general population.

\section{Patterns of myocardial fibrosis}

There is a growing body of literature showing that the phenotype of MF in athletes demonstrates large variance in patterns, location and quantification. More specifically, MF in athletes has been noticed to be divided into two main categories: non-ischemic and ischemic scar. These phenotypes seem to differ from that in the general population, which may be relevant for the underlying mechanisms and clinical prognosis of MF in apparently healthy athletes. As noted by Merghani et al. [45•], 14\% of veteran male athletes but none of the controls revealed LGE, half of whom had a pattern consistent with previous myocardial infarction. On the other hand, Tahir et al. [47••] demonstrated in 83 asymptomatic triathletes using LGE and ECV CMR focal non-ischemic MF in 9 of 54 (17\%) males but in none of the female triathletes. In lifelong veteran endurance athletes, MF has been reported near the septal insertion points of the RV free wall in $37 \%$ of the athletes with just one subject exhibiting sub-epicardial lateral wall fibrosis [51]. This phenotype seems to differ from that in the general population, which may be relevant for the underlying mechanisms and clinical prognosis of MF in apparently healthy athletes.

\section{A dose-response relationship?}

It is very well established that regular exercise improves cardiovascular risk profile, reduces the risk of myocardial infarction by $50 \%$ and is related with a variety of other beneficial effects. Most of these benefits are attributable to 
moderate exercise. Intense exercise, however, may infrequently trigger arrhythmogenic sudden cardiac death in asymptomatic cardiac disease. Moreover, long-standing vigorous exercise may be associated with adverse electrical and structural remodelling in otherwise normal hearts which may be a doseresponse phenomenon [52].

In parallel, evidence from case reports/series suggests that athletes diagnosed as having MF demonstrate high doses of exercise for many years. These studies and case reports suggest a dose-response relationship between lifetime exercise exposure and MF development [53]. Larger studies, such as by Tahir et al. $[47 \bullet \bullet]$, demonstrated focal non-ischemic MF in $17 \%$ males. A cycling race distance of $>1880 \mathrm{~km}$ completed during competition had the highest accuracy to predict LGE, with an area under the curve value of 0.876 , resulting in high sensitivity ( $89 \%)$ and specificity $(79 \%)$. Also, the swimming race distance was noted to be independent predictors of LGE presence. La Gerche et al. [54] studied 40 athletes at baseline, immediately following an endurance race (3$11 \mathrm{~h}$ duration) and 1-week post-race. LGE localized to the interventricular septum was identified in 5 of 39 athletes who had greater cumulative exercise exposure. Furthermore, in 108 apparently healthy male marathon runners with $\geq 5$ marathon competitions during the previous 3 years, the presence of LGE and of coronary artery calcification (CAC) in relation to cardiovascular risk factors was evaluated. It was noted that CAC percentile values and number of marathons independently predicted the presence of LGE [55].

In contrast, another report provided evidence of potential myocardial injury and ventricular dysfunction after prolonged exercise using CMR imaging before and after amateur marathon races in 28 healthy males. Although marathon running led to a transient increase of cardiac biomarkers, no detectable myocardial necrosis was observed as evidenced by LGE [56]. It is important to note that existing data is conflicting and the majority lacks direct verification of functional myocardial alterations by CMR especially in large number of athletes. Larger, well-designed studies, based on welldefined criteria of athletes' populations are needed in order to reach safe conclusions.

The presence of MF is an important risk factor for adverse cardiac outcomes in clinical populations. However, the prognostic value of MF has not been extensively studied in athletic population [51]. The impact of lifelong exercise training is still a matter of ongoing debate. Although there are data supporting a U-shaped association between exercise volume and cardiovascular risk, most available evidence suggests a curvilinear relationship, with greater health benefits at larger exercise doses [51, 57]. Several studies have identified the presence of LGE in the heart of extensively trained veteran athletes. La Gerche et al. [47••] have shown myocardial fibrosis by CMR and a reduction in RV systolic function in athletes with long-term exercise, suggesting that the heart has a limited capacity to tolerate the overload exercise. Some authors have also a new entity, the so called "Phidippides cardiomyopathy"; long-term strenuous exercise can induce cardiac dilation and also activates resident macrophages, pericytes and fibroblasts, resulting in the deposition of collagen and fibrosis. It may be possible that the RV is more susceptible to fatigue than the left ventricle after 
prolonged exercise although more studies are required to identify a probable effect of exercise "dose" and their implication in the development of heart failure [58].

In addition, nonischemic LV scar, which is characteristically localized at the mid-myocardial or subepicardial layers of the LV wall, can be found as mentioned above, in a broad spectrum of heart muscle diseases at risk of sudden cardiac death (SCD), including myocarditis, sarcoidosis, DCM, HCM and ARVC. Whether this isolated, segmental LV myocardial lesion may act as a substrate of life-threatening arrhythmias and SCD in the athlete remains to be elucidated. Also, the presence of LGE at the junction of the RV with the ventricular septum has been consistently reported in a sizeable proportion of endurance athletes and related to the duration and intensity of sports activity but this pattern is traditionally deemed a nonclinically relevant; however, follow-up studies evaluating its arrhythmic risk are lacking [53]. Zorzi et al. [59] evaluated 35 athletes with ventricular arrhythmias by CMR and compared with 40 healthy control athletes. A stria LGE pattern with subepicardial/midmyocardial distribution, mostly involving the lateral LV wall, was found in 27 (77\%) versus 0 in controls, whereas a spotty pattern of LGE localized at the junction of the right ventricle to the septum was, respectively, observed in 11 (31\%) versus 10 $(25 \%)$ though this did not reach statistical significance.

It is important to emphasize that the prognostic significance of nonspecific MF patterns seen in athletes is yet to be clarified as there is no significant evidence currently, that athletes with this pattern should be restricted from exercise.

\section{Conclusions}

Multimodality cardiac imaging has been used in all of its forms to study the intrigues of the athlete's heart syndrome. A description of the changes in cardiac morphology and function has permitted this syndrome to be differentiated from serious cardiac pathology, which may mimic it in many cases. Cardiac imaging has not successfully and conclusively answered this question, although recent advances in CMR who great promise. MF has been reported in endurance athletes and the pattern of LGE is heterogeneous, which may represent different causes and prognostic significance. Larger, well-designed studies, based on welldefined criteria of athletes' populations are needed in order to reach safe conclusions.

\section{Compliance with Ethical Standards}

\section{Conflict of Interest}

Emmanuel Androulakis and Peter P Swoboda each declare no potential conflicts of interest.

Human and Animal Rights and Informed Consent

This article does not contain any studies with human or animal subjects performed by any of the authors. 


\section{Open Access}

This article is distributed under the terms of the Creative Commons Attribution 4.0 International License (http://creativecommons.org/licenses/by/4.0/), which permits unrestricted use, distribution, and reproduction in any medium, provided you give appropriate credit to the original author(s) and the source, provide a link to the Creative Commons license, and indicate if changes were made.

\section{References and Recommended Reading}

Papers of particular interest, published recently, have been highlighted as:

- Of importance

$\bullet \quad$ Of major importance

1. Pluim BM, Zwinderman AH, van der Laarse A, van der Wall EE. The athlete's heart: a meta-analysis of cardiac structure and function. Circulation. 2000;101:336-44.

2. Sharma S, Drezner JA, Baggish A, Papadakis M, Wilson MG, Prutkin JM, et al. International recommendations for electrocardiographic interpretation in athletes. J Am Coll Cardiol. 2017;69:1057-75.

3. Utomi V, Oxborough D, Whyte GP, Somauroo J, Sharma S, Shave R, et al. Systematic review and metaanalysis of training mode, imaging modality and body size influences on the morphology and function of the male athlete's heart. Heart. 2013;99:1727-33.

4. Finocchiaro G, Papadakis M, Robertus JL, Dhutia H, Steriotis AK, Tome M, et al. Etiology of Sudden Death in Sports: Insights From a United Kingdom Regional Registry. J Am Coll Cardiol. 2016;67:2108-15.

5. La Gerche A. Can intense endurance exercise cause myocardial damage and fibrosis? Curr Sports Med Rep. 2013;12:63-9.

6. Goudis CA, Ntalas IV, Ketikoglou DG. Atrial fibrillation in athletes. Cardiol Rev. 2015;23:247-51.

7. Hinojar R, Botnar R, Kaski JC, Prasad S, Nagel E, Puntmann VO. Individualized cardiovascular risk assessment by cardiovascular magnetic resonance. Futur Cardiol. 2014;10:273-89.

8. American College of Cardiology Foundation Task Force on Expert Consensus Documents, Hundley WG, Bluemke DA, Finn JP, Flamm SD, Fogel MA, et al. ACCF/ACR/AHA/NASCI/SCMR 2010 expert consensus document on cardiovascular magnetic resonance: a report of the American College of Cardiology Foundation Task Force on Expert Consensus Documents. Circulation. 2010;121(22):2462-508.

9. La Gerche A, Taylor AJ, Prior DL. Athlete's heart: the potential for multimodality imaging to address the critical remaining questions. JACC Cardiovasc Imaging 2009; 2: 350-363.

10. Scharhag J, Schneider G, Urhausen A, Rochette V Kramann B, Kindermann W. Athlete's heart: right and left ventricular mass and function in male endurance athletes and untrained individuals determined by magnetic resonance imaging. J Am Coll Cardiol. 2002;40:1856-63.

11. Arbab-Zadeh A, Perhonen M, Howden E, Peshock RM, Zhang R, Adams-Huet B, et al. Cardiac remodeling in response to 1 year of intensive endurance training. Circulation. 2014;130:2152-61.

Demonstrated that one year of prolonged and intensive endurance training leads to cardiac morphological adaptations in 12 previously sedentary young subjects

12. Maron BJ, Doerer JJ, Haas TS, Tierney DM, Mueller FO. Sudden deaths in young competitive athletes: analysis of 1866 deaths in the United States, 1980-2006. Circulation. 2009;119:1085-92.

13. Rickers C, Wilke NM, Jerosch-Herold M, Casey SA, Panse P, Panse N, et al. Utility of cardiac magnetic resonance imaging in the diagnosis of hypertrophic cardiomyopathy. Circulation. 2005;112:855-61.

14. Moon JC, Mogensen J, Elliott PM, Smith GC, Elkington AG, Prasad SK, et al. Myocardial late gadolinium enhancement cardiovascular magnetic resonance in hypertrophic cardiomyopathy caused by mutations in troponin I. Heart. 2005;91:1036-40.

15. Nicholls M. The 2014 ESC Guidelines on the Diagnosis and Management of Hypertrophic Cardiomyopathy have been published. Eur Heart J. 2014;35:2849-50.

16. Petersen SE, Selvanayagam JB, Francis JM, Myerson SG, Wiesmann F, Robson MD, et al. Differentiation of athlete's heart from pathological forms of cardiac hypertrophy by means of geometric indices derived from cardiovascularmagnetic resonance. J Cardiovasc Magn Reson. 2005;7:551-8.

17. Scharf M, Brem MH, Wilhelm M, Schoepf UJ, Uder M, Lell MM. Atrial and ventricular functional and structural adaptations of the heart in elite triathletes assessed with cardiac MR imaging. Radiology. 2010;257:71-9. 
18. Caselli S, Maron MS, Urbano-Moral JA, Pandian NG, Maron BJ, Pelliccia A. Differentiating left ventricular hypertrophy in athletes from that in patients with hypertrophic cardiomyopathy. Am J Cardiol. 2014;114:1383-9.

19. Weng Z, Yao J, Chan RH, He J, Yang X, Zhou Y, et al. Prognostic Value of LGE-CMR in HCM: A Meta-Analysis. JACC Cardiovasc Imaging. 2016;9:1392-402.

20. Sheikh N, Papadakis M, Schnell F, Panoulas V, Malhotra A, Wilson M, et al. Clinical profile of athletes with hypertrophic cardiomyopathy. Circ Cardiovasc Imaging. 2015;8:e003454.

21. McDiarmid AK, Swoboda PP, Erhayiem B, Lancaster RE, Lyall GK, Broadbent DA, et al. Athletic cardiac adaptation in males is a consequence of elevated myocyte mass. Circ Cardiovasc Imaging. 2016;9:e003579.

22.• Swoboda PP, McDiarmid AK, Erhayiem B, Broadbent DA, Dobson LE, Garg P, et al. Assessing myocardial extracellular volume by $\mathrm{t} 1$ mapping to distinguish hypertrophic cardiomyopathy from athlete's heart. J Am Coll Cardiol. 2016;67:2189-90.

Demonstrated that CMR using T1 mapping has a potential role in the exclusion of HCM in athletes presenting with $\mathrm{LV}$ hypertrophy

23. Marcus FI, McKenna WJ, Sherrill D, Basso C, Bauce B, Bluemke DA, et al. Diagnosis of arrhythmogenic right ventricular cardiomyopathy/dysplasia proposed modification of the task force criteria. Circulation. 2010;121:1533-41.

24. Dalal D, Tandri H, Judge DP, Amat N, Macedo R, Jain $\mathrm{R}$, et al. Morphologic variants of familial arrhythmogenic right ventricular dysplasia/ cardiomyopathy a genetics-magnetic resonance imaging correlation study. J Am Coll Cardiol. 2009;53:1289-99.

25. te Riele AS, Tandri H, Bluemke DA. Arrhythmogenic right ventricular cardiomyopathy (ARVC): cardiovascular magnetic resonance update. J Cardiovasc Magn Reson. 2014;16:50.

26. Heidbüchel H, Hoogsteen J, Fagar R, Vanhees L, Ector $\mathrm{H}$, Willems R, et al. High prevalence of righ ventricular involvement in endurance athletes with ventricular arrhythmias Role of an electrophysiologic study I risk stratification. Eur Heart J. 2003;24:1473-80.

27. Kirchhof P, Fabritz L, Zwiener M, Witt H, Schäfers M, Zellerhoff S, et al. Age- and training-dependent development of arrhythmogenic right ventricular cardiomyopathy heterozygous plakoglobin-deficient mice. Circulation. 2006;114:1799-806.

28. D'Andrea A, Caso P, Severino S, Sarubbi B, Forni A, Cice $\mathrm{G}$, et al. Different involvement of right ventricular myocardial function in either physiologic or pathologic left ventricular hypertrophy: a Doppler tissue study. J Am Soc Echocardiogr. 2003;16:154-61.

29. La Gerche A, Claessen G, Dymarkowski S, Voigt JU, De Buck F, Vanhees L, et al. Exercise-induced right ventricular dysfunction is associated with ventricular arrhythmias in endurance athletes. Eur Heart J. 2015;36:1998-2010.
Demonstrated among athletes with normal cardiac function at rest that exercise testing can reveal RV contractile dysfunction among athletes with RV arrhythmias

30. Kuruvilla S, Adenaw N, Katwal AB, Lipinski MJ, Kramer $\mathrm{CM}$, Salerno M. Late gadolinium enhancement on cardiac magnetic resonance predicts adverse cardiovascular outcomes in nonischemic cardiomyopathy: a systematic review and meta-analysis. Circ Cardiovasc Imaging. 2014;7:250-8.

31. Sandstede J, Lipke C, Beer M, Hofmann S, Pabst T, Kenn W, et al. Age- and gender-specific differences in left and right ventricular cardiac function and mass determined by cine magnetic resonance imaging. Eur Radiol. 2000;10:438-42.

32. Assomull RG, Prasad SK, Lyne J, Smith G, Burman ED, Khan $\mathrm{M}$, et al. Cardiovascular magnetic resonance, fibrosis, and prognosis in dilated cardiomyopathy. J Am Coll Cardiol. 2006;48:1977-85.

33. Abergel E, Chatellier G, Hagege AA, Oblak A, Linhart A, Ducardonnet A, et al. Serial left ventricular adaptations in world-class professional cyclists: implications for disease screening and follow-up. J Am Coll Cardiol. 2004;44:144-9.

34. Abernethy WB, Choo JK, Hutter AM Jr. Echocardiographic characteristics of professional football players. J Am Coll Cardiol. 2003;41:280-4.

35. Claessen G, Schnell F, Bogaert J, Claeys M, Pattyn N, De Buck F, Dymarkowski S, Claus P, Carré F, Van Cleemput J, La Gerche A, Heidbuchel H. Exercise cardiac magnetic resonance to differentiate athlete's heart from structural heart disease. Eur Heart J Cardiovasc Imaging 2018.

36. Puntmann VO, Voigt T, Chen Z, Mayr M, Karim R, Rhode $\mathrm{K}$, et al. Native T1 mapping in differentiation of normal myocardium from diffuse disease in hypertrophic and dilated cardiomyopathy. JACC Cardiovasc Imaging. 2013;6:475-84.

37. Ugander M, Oki AJ, Hsu LY, Kellman P, Greiser A, Aletras AH, et al. Extracellular volume imaging by magnetic resonance imaging provides insights into overt and sub-clinical myocardial pathology. Eur Heart J. 2012;33:1268-78.

38. Mordi I, Carrick D, Bezerra H, Tzemos N. T1 and T2 mapping for early diagnosis of dilated nonischaemic cardiomyopathy in middle-aged patients and differentiation from normal physiological adaptation. Eur Heart J Cardiovasc Imaging. 2016;17:797-803.

39. Petersen SE, Selvanayagam JB, Wiesmann F, Robson MD, Francis JM, Anderson RH, et al. Left ventricular non-compaction: insights from cardiovascular magnetic resonance imaging. J Am Coll Cardiol. 2005;46:101-5.

40. Gati S, Sharma S. CardioPulse: the dilemmas in diagnosing left ventricular non-compaction in athletes. Eur Heart J. 2015;36:891-3.

41. Gati S, Chandra N, Bennett RL, Reed M, Kervio G, Panoulas VF, et al. Increased left ventricular trabeculation in highly trained athletes: do we need 
more stringent criteria for the diagnosis of left ventricular non-compaction in athletes? Heart. 2013;99:401-8.

42. Caselli S, Ferreira D, Kanawati E, Di Paolo F, Pisicchio C, Attenhofer Jost C, et al. Prominent left ventricular trabeculations in competitive athletes: A proposal for risk stratification and management. Int J Cardiol. 2016;223:590-5.

43. Ripley DP, Saha A, Teis A, Uddin A, Bijsterveld P, Kidambi A, et al. The distribution and prognosis of anomalous coronary arteries identified by cardiovascular magnetic resonance: 15 year experience from two tertiary centres. J Cardiovasc Magn Reson. 2014;16:34.

44. Prakken NH, Cramer MJ, Olimulder MA, Agostoni P, Mali WP, Velthuis BK. Screening for proximal coronary artery anomalies with 3-dimensional MR coronary angiography. Int J Card Imaging. 2010;26:701-10.

45. Merghani A, Maestrini V, Rosmini S, Cox AT, Dhutia $\mathrm{H}$, Bastiaenan R, et al. Prevalence of subclinical coronary artery disease in masters endurance athletes with a low atherosclerotic risk profile. Circulation. 2017;136:126-37.

Veteran male athletes but none of the controls revealed LGE, half of whom had a pattern consistent with previous myocardial infarction

46. Breuckmann F, Möhlenkamp S, Nassenstein K, Lehmann N, Ladd S, Schmermund A, et al. Myocardial late gadolinium enhancement: prevalence, pattern, and prognostic relevance in marathon runners. Radiology. 2009;251:50-7.

47.• Tahir E, Starekova J, Muellerleile K, von Stritzky A, Münch J, Avanesov M, et al. Myocardial fibrosis in competitive triathletes detected by contrast-enhanced CMR correlates with exercise-induced hypertension and competition history. JACC Cardiovasc Imaging 2017.

In eighty-three asymptomatic triathletes non-ischaemic MF was found in $17 \%$ of males.

48. Sanchis-Gomar F, López-Ramón M, Alis R, Garatachea N, Pareja-Galeano H, Santos-Lozano A, et al. No evidence of adverse cardiac remodeling in former elite endurance athletes. Int J Cardiol. 2016;222:171-7.

49. Wilson M, O'Hanlon R, Prasad S, Deighan A, Macmillan P, Oxborough D, et al. Diverse patterns of myocardial fibrosis in lifelong, veteran endurance athletes. J Appl Physiol (1985). 2011;110:1622-6.

50. Schnell F, Claessen G, La Gerche A, Bogaert J, Lentz PA, Claus $\mathrm{P}$, et al. Subepicardial delayed gadolinium enhancement in asymptomatic athletes: let sleeping dogs lie? Br J Sports Med. 2016;50:111-7.

51. Eijsvogels TM, Oxborough DL, O'Hanlon R, Sharma S, Prasad S, Whyte G, et al. Global and regional cardiac function in lifelong endurance athletes with and without myocardial fibrosis. Eur J Sport Sci. 2017;17:1297-303.

52. Sharma S, Merghani A, Mont L. Exercise and the heart: the good, the bad, and the ugly. Eur Heart J. 2015;36:1445-53.

53. van de Schoor FR, Aengevaeren VL, Hopman MT, Oxborough DL, George KP, Thompson PD, et al. Myocardial fibrosis in athletes. Mayo Clin Proc. 2016;91:1617-31.

54. La Gerche A, Burns AT, Mooney DJ, Inder WJ, Taylor AJ, Bogaert J, et al. Exercise-induced right ventricular dysfunction and structural remodeling in endurance athletes. Eur Heart J. 2012;33:998-1006.

55. Möhlenkamp S, Lehmann N, Breuckmann F, BröckerPreuss M, Nassenstein K, Halle M, et al. Running: the risk of coronary events: Prevalence and prognostic relevance of coronary atherosclerosis in marathon runners. Eur Heart J. 2008;29:1903-10.

56. Hanssen H, Keithahn A, Hertel G, Drexel V, Stern H, Schuster T, et al. Magnetic resonance imaging of myocardial injury and ventricular torsion after marathon running. Clin Sci (Lond). 2011;120:143-52.

57. Arem H, Moore SC, Patel A, Hartge P, Berrington de Gonzalez A, Visvanathan K, et al. Leisure time physical activity and mortality: a detailed pooled analysis of the dose-response relationship. JAMA Intern Med. 2015;175:959-67.

58. Carbone A, D'Andrea A, Riegler L, Scarafile R, Pezzullo $\mathrm{E}$, Martone F, et al. Cardiac damage in athlete's heart: When the "supernormal" heart fails! World J Cardiol. 2017;9:470-80.

59. Zorzi A, Perazzolo Marra M, Rigato I, De Lazzari M, Susana A, Niero A, et al. Nonischemic left ventricular scar as a substrate of life-threatening ventricular arrhythmias and sudden cardiac death in competitive athletes. Circ Arrhythm Electrophysiol 2016; 9. 